\title{
DE L'INTÉRET PISCICOLE DES RETENUES HYDRO-ÉLECTRIQUES
}

\author{
par Roger BACHELIER \\ Inspecteur des Eaux et Forêts
}

II n'y a guère qu'une trentaine d'années que les premières retenues hydro-électriques ont été aménagées pour produire de "l'électricité de pointe ". Depuis, elles ont été multipliées et elles le seront encore non seulement pour faire face aux " pointes journalières " mais aussi aux " pointes saisonnières", compte tenu des conditions climatiques.

Relativement aux 100.000 hectares d'étangs exploités en France les dizaines de milliers d'hectares de retenue réalisés ou projetés méritent de ne pas être négligés au point de vue piscicole, malgré leur situation souvent excentrique et leur capacité biogénique toujours faible.

\section{Les inConvénients de ces Retenues aU poInt de vue piscrcole.}

Tous les pêcheurs de Truites ou de Saumons sont opposés à la constitution de ces retenues, de même qu'à l'édification de tout barrage élevé, parce qu'elles nuisent ou même font disparaittre le peuplement en Salmonidés des cours d'eau où elles sont aménagées.

S'il s'agit d'un peuplement de Saumons, le dommage est toujours considérable et parfois irrémédiable. La richesse d'un fleuve en Saumons dépend essentiellement de ses possibilités en frayères et de sa capacité biogénique pour le Tacon puisque le Tacon devient Saumon dans l'Atlantique. L'Inspecteur Vibert estime qu'il faut compter 20 Tacons de 40 grammes pour permettre la remontée d'un Saumon de 8 kilogrammes en moyenne; dans ces conditions, si une rivière est capable de nourrir un certain poids de Salmonidés et que ceux-ci soient des Tacons, Ies pêcheurs y auront à leur disposition un poids dix fois supérieur de Saumons, indépendamment de l'attrait exceptionnel de la pêche du Saumon et de sa valeur vénale double de celle de la Truite.

L'édification d'un seul barrage infranchissable à l'aval de la zone des frayères fait disparaître tout le Saumon d'une rivière. Un tel anéantissement brusque fut assez rare parce que les premiers grands barrages furent construits dans les hautes vallées et n'isolaient que la partie supérieure des zones frayères (Pouthès dans l'Allier); tandis que les barrages édifiés à l'aval des frayères, en région non montagneuse, n'étaient pas totalement infranchissables (Decize, sur la Loire; Châtellerault, sur la Vienne). Dans l'un comme dans l'autre cas, les pêcheurs qui profitaient de la stagnation des Saumons au pied des barrages, ne se rendaient pas 
bien compte du dommage insidieux que ces barrages leurs causaient, et pourtant ceux-ci diminuaient soit le nombre des frayères, soit le nombre des géniteurs pouvant atteindre les frayères.

Un barrage de retenue dans la zone des frayères non seulement interdit au Saumon l'accès de ses frayères d'amont, mais, alimentant une usine fonctionnant "par éclusées ", il compromet dangereusement la fertilité des frayères d'aval à une distance plus ou moins grande suivant l'ampleur des éclusées : des pontes mises à sec quelques heures chaque jour ou quelques jours chaque semaine ont, en effet, peu de chance d'éclore.

Pour la Truite, dont la ponte est moins profonde, les assecs périodiques d'aval sont encore plus néfastes. Par contre, le barrage ne nuit pas à son peuplement á l'amont de la retenue; on pourrait même dire qu'il le favorise par la disparition des Tacons qui le concurrençaient. Sans doute, les Truites d'aval ne viennent plus sur les frayères à l'amont mais peu importe au pécheur, si ce n'est au braconnier.

Lorsqu'un barrage provoque une retenue, les Truites de la rivière noyée y grossissent démesurément et font la joie des pêcheurs..... jusqu'au jour où elles disparaissent pour faire place à des poissons blancs peu nombreux et peu intéressants. Et cette disparition de la Truite peut même se produire à l'aval de la restitution malgré les déversements d'alevins compensant la disparition des frayères d'amont et la stérilité des frayères d'aval.

La disparition de la Truite est due à la désoxygénation de l'eau dans la retenue, au moins en été, sous le double effet de l'échauffement de l'eau ensoleillée et de la fermentation de la vase accumulée. (Ce double effet de désoxygénation peut être compensé dans les étangs par l'assimilation chlorophylienne des plantes aquatiques, mais il ne peut en être ainsi dans les retenues hydro-électriques où les variations de niveau ne permettent pas l'installation d'une végétation aquatique suffisante.)

Cet inconvénient, inexistant à grande altitudẹe, est fréquent dans les régions mi-montagneuses où la pêche est beaucoup pius pratiquée, et il ne doit pas être négligé lorsque l'on envisage la construction d'un barrage de compensation ; celui-ci désoxygènera encore l'eau dont il est chargé de régulariser le débit.

Que le barrage constitue une simple prise d'eau ou une retenue, s'il alimente une usine par une dérivation, le lit de la rivière entre le barrage et la restitution ne sera plus mouillé que lors des crues. Théoriquement, un débit constant doit être réservé à travers le barrage, mais lorsqu'il est respecté, il est généralement trop faible pour donner à la " vieille rivière " méme l'aspect d'un ruisseau. Lorsqu'une crue se déverse par dessus le barrage dans la "vieille rivière ", ce qui normalement est plus fréquent à l'époque de la fraye des Salmonidés, les Truites vivant à l'aval de la restitution s'engagent dans la "vieille rivière ", risquant fort d'être surprises par un assec brusque qui peut leur être fatal avec ou sans braconnage. Cette brusquerie de l'assec d'une "vieille rivière "est presque normale du fait que le débit dérivé dans l'usine est constant lors d'une crue et au moins égal au débit moyen, si bien qu'un débit dans la "vieille rivière " correspond seulement à une pointe de crue généralement crois- 
sante et décroissante rapidement. D'autre part, si les Truites ont eu le temps de pondre dans la "vieille rivière ", leur ponte est irrémédiablement condamnée.

Le risque de mortalité des Salmonidés et de leurs pontes dans les vieilles rivières est d'autant plus grand que les déversements au barrage sont plus fréquents, à moins qu'une échelle à poissons efficace ait été aménagée dans le barrage. Il existe et on construit encore des retenues tellement vastes que celles-ci emmagasinent les crues sans déverser; dans ce cas particulier, la "vieille rivière" est sans danger.

\section{LeS COMPENSATIONS AUX DOMMAGES PISCICOLES.}

Pour compenser les dommages causés à la reproduction des poissons migrateurs, l'Administration impose aux concessionnaires de barrage de déverser, chaque année, des alevins de Truite en nombre calculé d'après la formule du Professeur LÉGER. Cette compensation n'est souvent qu'un pis aller en raison des modifications apportées par le barrage et la retenue aux conditions de vie dans le cours d'eau. Faute de pouvoir maintenir le peuplement primitif, mieux vaudrait assurer un peuplement rationnel dans la retenue, la pêche n'y serait plus la même que dans la rivière noyée, mais elle y serait néamnoins attractive et éventuellement lucrative.

Vouga vient de publier une étude très intéressante à ce sujet "Aménagement piscicole d'un lac de barrage». Sans doute est-ce volontairement qu'il a employé le terme de "lac", c'est-à-dire qu'il a considéré la retenue de Rossens comme si elle devait se comporter comme un lac si ce n'est la variation du niveau de l'eau. Les lacs, du fait même de leur existence plusieurs fois millénaires, ne se comblent pas ni même ne s'envasent d'une manière appréciable. L'étude des matériaux chariés par les affluents du "Lac de Rossens" permet peut-ètre de supposer que cette retenue ne s'envasera pas. Pour notre part, nous ne connaissons pas encore une retenue à l'abri de ce gros inconvénient et mème dans les retenues élevées, où la fraîcheur de l'eau peut compenser l'action réductrice de la vase, nous imaginons mal des frayères de Salmonidés, quels qu'ils soient, dans le périmètre entièrement vaseux de la retenue.

Dans le cas où l'altitude de la retenue est suffisamment élevée ou son envasement si faible que la Truite s'y maintienne, nul doute que l'alevinage imposé au concessionnaire soit efficace, mais il est malheureusement insuffisant car il a été calculé pour peupler la rivière noyée dont la capacité biogénique totale était forcément inférieure à celle de la retenue, malgré l'insignifiance de sa végétation.

Sì l'eau de la retenue est insuffisamment oxygénée pour la Truite, il est évident que d'y déverser de faibles alevins particulièrement exigeants en oxygène constitue un vain gaspillage, et il peut même en être de même à l'aval de la restitution si la rivière à Truites est devenue une rivière à poissons blancs. Dans ce cas, nous verrons par la suite qu'il serait tout à fait indiqué d'aleviner en poissons blancs y compris des Brochets et ainsi, pour n'être plus sportive, la pêche dans la retenue n'en serait pas moins intéressante. 


\section{LES VIDANGES DE RETENUE.}

Vouga n'a pas envisagé une éventualité de plus en plus fréquente en France et fort importante au point de vue piscicole : la vidange de la retenue. Des personnalités compétentes de l'Électricité de France estiment que l'entretien des barrages et des prises d'eau exigerait la vidange totale toutes les dizaines d'années en moyenne, principe qui n'a pas été respecté jusqu'à présent du fait de l'exploitation par Sociétés privées et de la pénurie d'électricité, mais que l'on peut supposer devoir être retenu un jour par l'Électricité de France.

L'article 23 du décret du 29 Août 1939 prescrit : a Il est interdit de pêcher dans les parties de cours d'eau, canaux ou RÉSERvoIrs dont le niveau est abaissé artificiellement... Si les Services de la police de la pẻche l'estiment nécessaire en vue d'assurer la protection du poisson, les Préfets peuvent prescrire aux intéressés d'évacuer et de transporter dans l'eau libre qui leur sera désignée, les poissons retenus devant les ouvrages ou mis en danger par les manœuvres d'eau effectuées ".

D'autre part, le Cahier des Charges du Règlement d'eau du barrage prescrit parfois au concessionnaire d'aviser la Gendarmerie et les Eaux et Forêts quarante-huit heures à l'avance de la vidange de la retenue.

En ce qui concerne l'Électricité de France, chaque fois qu'elle estime qu'il s'agit d'un barrage de retenue et non d'un barrage de prise d'eau, appréciation qu'il y aurait lieu de préciser, elle prévient régulièrement les Eaux et Forêts et la Gendarmerie, qui envoient leurs agents assister à la vidange pour empêcher le braconnage du poisson. Parfois les Eaux et Forêts, en accord avec les Ponts et Chaussées, provoquent un arrêté préfectoral prescrivant certaines mesures pour la protection du poisson telles que vitesse de vidange, fermeture nocturne partielle, fermeture progressive du barrage, etc...

Nous ne nous étendrons pas sur les résultats habituels des vidanges si ce n'est que les Sociétés de pêche accusent le concessionnaire d'avoir fait périr des tonnes de poissons et parfois lui demandent des dommages basés sur la valeur du dit poisson.

Cependant, nous retiendrons les observations que nous avons faites au cours des quelques vidanges auxquelles nous avons pu assister et nous les classerons en deux catégories : les inconvénients et les intérêts des vidanges.

\section{Inconvénients des vidanges.}

$1^{\circ}$ Époque défavorable. - Les conditions d'exploitation hydro-électrique, commandées en partie par une production d'électricité insuffisante, obligent le plus souvent à vidanger totalement les retenues en été. A cette époque, le poisson est beaucoup plus fragile. Même en le transportant dans une eau rafraîchie artificiellement, il résiste beaucoup moins qu'en hiver car il n'est pas en léthargie; les Carpes elles-mẻmes sont difficiles à conserver. Il est bien certain qu'une même retenue vidangée à la même vitesse en hiver causerait beaucoup moins de mortalité et même peut être aucune. Il y a donc grand intérèt à persuader le conces- 
sionnaire de vidanger le plus tôt possible au printemps ou le plus tard possible en automne.

$2^{\circ}$ Vitesse trop rapide. - Les prises d'eau dans les retenues sont toujours situées à quelques mètres au-dessus du fond même lorsqu'il n'y a aucun risque de comblement, sans doute pour n'envoyer dans les turbines que de l'eau décantée. La masse d'eau située au-dessous de la prise d'eau constitue un " matelas " non turbinable. Généralement, le concessionnaire peut turbiner lentement sans perte d'énergie tout le volume " utile ", mais lorsqu'il ne reste plus que le matelas, il a tout avantage à vidanger rapidement pour hâter les réparations du barrage et la remise en eau. Cette vidange rapide en période chaude cause généralement la mortalité du poisson, l'abaissement rapide de l'eau brassant la vase qui désoxygène l'eau et mème colmate les ouïes des poissons ayant pu résister à la désoxygénation de l'eau.

Les intérêts du concessionnaire sont opposés aux intérêts piscicoles; pour satisfaire ces derniers, le matelas ne saurait être vidangé trop lentement, tandis qu'il importe peu que le volume habituellement turbiné le soit rapidement.

Pour essayer de trouver un compromis, nous avions préconisé une vidange du matelas de plus en plus lente; ce faisant nous avions commis deux erreurs :

Ce n'est pas tant le "matelas" qui doit être vidangé lentement, mais tout le volume de la retenue qui n'est pas turbiné habituellement; c'est ainsi que dans les retenues alimentant des usines " de pied de barrage", la tranche d'eau normalement turbinée est relativement très faible; dans ce cas c'est la quasi-totalité de la retenue qui devrait être vidangée lentement. D'autre part, nous avions pensé que l'épaisseur de vase était sensiblement proportionnelle à la profondeur, tel qu'il apparaît dans les étangs, mais généralement les retenues hydro-électriques sont fort longues et la plupart des matières en suspension dans l'eau se sont déposées bien avant que celle-ci ne s'engage dans la prise d'eau, Enfin, il peut y avoir une assez grande distance entre la prise d'eau et le barrage, zone dans laquelle l'eau n'étant pas renouvelée, il n'y a que peu de décantation. Le maximum d'épaisseur de la vase peut donc se trouver à l'amont de la retenue; cette localisation dépend des variations de section de la retenue et de la vitesse d'écoulement dans celle-ci.

Pour réduire au minimum les risques de mortalité dus à la vidange trop rapide, il faut tenir compte du profil en long de la retenue entre la - cote de fléchissement " (le niveau inférieur de la tranche turbinée habituellement) et l'aplomb de la prise d'eau ; et du délai maximum accepté par le concessionnaire pour vidanger. La vitesse d'abaissement du niveau de l'eau devrait être proportionnelle à l'inclinaison du profil en long de façon à ce que l'exondation soit sensiblement constante.

Il n'y a pas lieu de considérer les deux ou trois derniers mètres de la profondeur; à ce niveau la vidange est quasi terminée et il faut, au contraire, l'accélérer au maximum pour que le poisson encore en vie puisse survivre.

Nous n'avons pas indiqué de vitesse optima de vidange car celle-ci ne 
saurait jamais ètre trop petite sous réserve de n'être pas négative (un relèvement du niveau en cours de vidange est, en effet, tout à fait à proscrire puisqu'il provoquerait un deuxième brassage de la vase). Si le concessionnaire voulait bien être assez patient, il faudrait s'efforcer de limiter la vitesse d'abaissement de niveau à 1 ou 2 centimètres à l'heure.

$3^{\circ}$ Permanence de l'arrivée d'eau. - A première vue une arrivée d'eau permanente à l'amont semblerait un excellent remède à la désoxygénation de l'eau d'une retenue en vidange. En réalité, cette arrivée d'eau remet en mouvement la totalité de la vase déposée dans l'ancien lit de la rivière, et, en fait d'eau fraîche, c'est du "café " qui arrive dans une tasse de "café au lait". Si la cote de flèchissement de la retenue était très basse et si son matelas était faible, il pourrait ne plus en être de même, mais c'est un cas assez rare.

L'équipement hydro-électrique de nos rivières est déjà tellement avancé qu'il est fréquent qu'une retenue en vidange soit précédée par une autre retenue à l'amont ; dans ce cas, il serait souhaitable d'abaisser simultanément les deux retenues puis de fermer la retenue amont dès qu'elle serait capable d'emmagasiner tout le débit de la rivière jusqu'à la fin de la vidange totale de la retenue aval.

\section{Intérêts des vidanges.}

Certains pècheurs, qui s'indignaient violemment de la "catastrophe " causée par la vidange de la retenue où ils allaient habituellement pêcher, reconnaissent avec surprise, par la suite, que leur pêche non seulement s'est reconstituée mais s'est améliorée.

La vidange est la façon culturale de base de tous les étangs; pourquoi n'en serait-il pas de mẻme pour les retenues hydro-électriques? Malheureusement pour celles-ci, on ne peut pas envisager un assec hivernal régulier, mais une seule vidange élimine l'eau de profondeur souvent désoxygénée (le Service des Recherches Piscicoles a constatć en Septembre l'absence d'oxygène à partir de 6 mètres de fond dans le barrage d'Éguzon), une quantité très appréciable de vase, "et tout le poisson " s'écrieront les pêcheurs..., sans doute, mais quel poisson? Quelques vieillards géants (donc cannibales) des espèces qui se reproduisent peu ou pas dans la retenue, et des nains des espèces qui, se reproduisant, sont trop nombreux par rapport à la capacilé biogénique de la retenue, sans parler éventuellement des Poissons-Chats ou des Perches-Soleils.

Le repeuplement naturel par la rivière, complété par un alevinage rationnel qui ne risquera plus d'être la proie des vieillards géants éliminés, dédommagera largement les pêcheurs de l'année de mise en réserve qu'ils auront dù respecter après la vidange.

L'alevinage rationnel dépend de la capacité biogénique de la retenue, du peuplement existant dans la rivière à l'amont de la retenue ou subsistant dans celle-ci après la vidange, et enfin du goût de la majorité des pêcheurs.

Au lieu d'être un sujet de scandale les vidanges de retenues pourraient être une source de profit et d'amélioration piscicole; nous allons voir de quelle façon. 
Possibilutés d'avenir piscicole des retenues hydro-Électriques.

L'exploitation piscicole des retenues hydro-électriques n'a pratiquement jamais été envisagée, ni par le législateur, ni par les concessionnaires. Nous examinerons successivement les mesures réglementaires et les aménagements de construction nécessaires ou tout au moins favorables à cette exploitation.

\section{I. - Mesures réglementaires.}

Comme nous l'avons vu, les retenues hydro-électriques sont considérées comme des eaux libres. De ce fait, lors des vidanges : ou aucune mesure n'a été prévue et tout le poisson doit être abandonné, qu'il soit vif ou mort; ou le Préfet a prescrit par arrêté aux intéressés "d'évacuer et de transporter dans l'eau libre qui leur sera désignée, les poissons retenus devant les ouvrages ou mis en danger par les manœuvres d'eau effectuées ".

Concessionnaire ou Société de pêche sont aussi incapables les uns que les autres de récupérer tout le poisson vivant d'une retenue pour le remettre en eau libre; d'autre part, nous avons vu de quelles sortes de poisson il s'agit. Dans quelle eau libre serait-il souhaitable de le remettre?

Enfin, en supposant qu'il soit possible de transporter le poisson de la retenue dans une eau libre, la reprise de celui-ci et son transport seront - très onéreux et sans aucun intérêt pour le concessionnaire (ou le titulaire du droit de pèche) qui, après la vidange, devra encore faire les frais d'un alevinage.

Le décret du 5 Septembre 1897 était moins strict que celui du 29 Août 1939 puisqu'il permettait au Préfet d'autoriser le titulaire du droit de pêche à se servir d'engins n'ayant pas les dimensions réglementaires pour " s'emparer " du poisson menacé de périr lors de vidange des biefs, biefs dont le peuplement piscicole était, à l'époque, tout à fait analogue à celui de la rivière barrée.

Nous ne serions pas d'avis de voir remettre ce texte en vigueur pour les simples biefs qui correspondent à des prises d'eau, mais il pourrait l'être pour les retenues dont le peuplement est nettement différent de celui de la rivière. (Ces retenues pourraient ètre définies par un volume supérieur ou égal à 80.000 fois le module de la rivière barrée, par exemple, c'est-à-dire capable de retenir le débit moyen de la rivière pendant près d'une journée. De cette façon, le titulaire du droit de pêche, quel qu'il soit, pourrait être tenté par la rentabilité de l'opération consistant à reprendre tout le poisson de la retenue lors de la vidange, mème si cette reprise était subordonnée à une charge d'alevinage rationnel. Ainsi, non seulement le poisson ne serait plus perdu ou braconné, ce qui constitue toujours un exemple regrettable, mais la valeur piscicole de la retenue serait encore très améliorée.

Un moyen pour obtenir ce résultat dans le cadre des règlements actuels consisterait à obtenir la "clôture " de la retenue. La clôture côté aval d'une retenue est dèjà réalisée au point de vue pratique et réglementaire. 
puisque le règlement d'eau du barrage impose généralement des charges piscicoles pour compenser l'interruption apportée à la circulation du poisson, mais il n'en est pas de même à l'amont où il faut réglementairement une grille assez fine. Sans doute celle-ci est un leurre car si elle empêche les poissons de la retenue d'aller frayer dans l'affluent elle n'empèche pas les alevins de cet afluent de descendre dans la retenue; cependant, il semble assez difficile d'envisager une modification de la réglementation de la clôture à ce sujet. Peut être cette modification serait-elle envisageable au bénéfice des retenues telles que nous avons proposé de les définir ci-dessus et dont le peuplement diffère généralement tout à fait de celui de l'afluent; mais ce ne peut être qu'un vou et des retenues seront encore vidangées l'été prochain.

Il existe des retenues où la pose de grilles à l'amont peut etre réalisée au simple point de vue piscicole; ce sont les retenues peuplées de Truites. Comme l'a indiqué VougA dans son article "Aménagement piscicole d'un lac de barrage ": de telles retenues méritent la construction de pièges fixes à l'amont de la retenue pour récolter les oufs des géniteurs au moment de la fraye. La petite expérience que nous avons de ce genre de reprise de géniteurs nous laisse croire que celle-ci n'est au mieux que très partielle, mais, faute de pouvoir vidanger la retenue, c'est néanmoins le moyen le plus pratique de se procurer des cufs.

La construction d'un piège comportant obligatoirement des grilles représente d'ailleurs un faible investissement doublement rentable pour le propriétaire ayant acquis le droit de clore sa retenue : chaque année, il récolte des œufs de Truite sauvage très appréciés et, lors de la vidange, tout le poisson lui appartient. Les seules sujétions de cette clôture amont sont la surveillance et le défeuillage; cependant, la surveillance d'une eau close ne relevant pas de la police de la pêche, le gardien du barrage est parfaitement habilité pour l'exercer sans formalités; d'autre part, la grande sujétion du défeuillage peut être évitée par l'utilisation de grilles horizontales ou mieux, tournantes, celles-ci se défeuillant d'ellesmêmes.

\section{II. - Aménagements piscicoles des retenues.}

Il y a deux sortes d'aménagements à envisager :

10 Ceux tendant à améliorer la production et la reproduction du poisson ;

$2^{\circ}$ Ceux permettant la reprise du poisson et évitant la mortalité lors des vidanges.

\section{Améliorations de la pRoduction.}

a) Par action sur le milieu.

La principale consisterait à diminuer dans la mesure du possible les variations de niveau d'eau. Il ne peut être question de vouloir modifier le régime de marche de l'usine, raison d'être de la retenue; mais souvent la retenue est coupée par un pont nouveau, ou elle recouvre à peine une digue ou un talus, ces ouvrages modifiés en conséquence peuvent per- 
mettre une diminution des variations de niveau à leur amont. Évidemment, on ne pourrait obtenir du concessionnaire de renoncer totalement à l'utilisation de l'eau retenue par ce pont ou par cette digue, mais en obtenant que celle-ci ne soit utilisée qu'en cas de besoin, cette retenue partielle pourrait être exploitée sensiblement comme un étang et produire du poisson en conséquence. C'est, en effet, avant les pluies d'automne ou avant la fonte des neiges au printemps que le concessionnaire est généralement amené à utiliser la totalité de la " tranche utile ", époque justement favorable à la pêche par vidange de la retenue partielle.

La désoxygénation de l'eau de la retenue, du fait de la vase, élimine souvent la population naturelle de Truites et peut même réduire l'espace vital des Cyprinides lorsqu'elle est très poussée. Un moyen pour lutter contre ce défaut, indépendamment des vidanges, consisterait à ouvrir les vannes de fond, notamment lorsque la retenue déverse, ce qui ne causerait aucune perte d'énergie. Non seulement ce dévasage améliorerait les conditions de vie dans la retenue, mais il rendrait moins néfastes les effets des vidanges ultérieures tant dans la retenue qu'à l'aval de celle-ci.

Un autre moyen d'évacuer l'eau de profondeur désoxygénée, si ce n'est la vase, est d'assurer l'évacuation du " débit réservé " par un orifice situé assez bas dans le barrage.

L'intérêt économique de l'utilisation d'engrais dans les étangs ne se discute pas plus que pour les terres de cultures, mais en raison de l'écoulement relativement rapide de l'eau dans les retenues hydro-électriques, nous aurions hésité à y conseiller des épandages d'engrais. Or, la Fédération de la Corse, faute de pouvoir se procurer des alevins, a tenté d'améliorer le peuplement de ses ruisseaux par des déversements d'engrais et a été très satisfaite du résultat.

En Corse, mais aussi dans les nombreuses retenues constituées en région granitique, l'insuffisance de la capacité biogénique des eaux est due à leur acidité ; sans doute serait-il peu rentable de vouloir atteindre artificiellement le degré hydrotimétrique optimum de $15^{\circ}$, mais pour des eaux ne titrant que quelques degrés hydrotimétriques, il est certain que des déversements de chaux, même relativement peu importants, auraient des effets très sensibles. D'autre part, les retenues hydro-électriques se succèdant souvent le long des rivières, il est bien certain que la chaux entraînée d'une retenue supérieure profitera à toute celles situées à son aval.

\section{b) Par l'exploitation de l'Anguille.}

De nombreux propriétaires d'étangs poursuivent encore l'exploitation de l'Anguille comme leur avait conseillè le regretté Inspecteur LE CLERc voici une trentaine d'années. Cet élevage s'accorde mal avec une exploitation normale d'étang qui comporte un assec périodique, mais justement tel n'est pas le cas des retenues.

Le grand reproche que les pêcheurs font à l'Anguille est de dévorer le frai. Sans doute est-ce vrai, mais nous avons vu quel était la fraie des retenues : pour certaines espèces, il n'y en a pas et, pour d'autres, il y en 
a de trop. Quant à nuire aux poissons éclos, nous n'avons jamais entendu de plaintes à ce sujet de la part des pisciculteurs élevant l'Anguille et les observations semblent prouver que l'Anguille est moins ichtyophage, si ce n'est carnivore, que le Brochet.

L'élevage de l'Anguille en retenue hydro-électrique aurait le double avantage d'utiliser au mieux la capacité biogénique du fond vaseux et de pouvoir récupérer la quasi-totalité de la production sans vidange. En effet, la réglementation actuelle permet de capturer les Anguilles d'avalaison dans les coursiers d'usine; or, toutes les Anguilles dévalent pour aller se reproduire dans la mer des Sargasses et il est toujours possible d'installer une nasșe de capture à l'amont ou à l'aval des turbines et, parfois aussi, dans le déversoir, d'autant plus que l'avalaison des Anguilles ne dure que deux ou trois mois d'automne.

Malgré ses mœurs quasi-amphibies, la Civelle ne remonte pas ou guère au-dessus des grands barrages et toute la production que l'on pourrait escompter dans les retenues devrait provenir de Civelles qui y seraient déversées. En étang, le chargement en Civelles est habituellement effectué sur la base de 200 à l'hectare. En retenue hydro-électrique, étant donné la remontée certaine d'une grande proportion de Civelles déversées dans les affluents de la retenue et le faible prix des Civelles (10 centimes pièce à l'heure actuelle), nous croyons qu'il pourrait en être déversé 500 à l'hectare, et ce n'est d'ailleurs que quatre ans après le premier déversement qu'il y aurait lieu d'en envisager la reprise au coursier de l'usine et, éventuellement, au déversoir. Reprise qui, naturellement, n'empêcherait pas tous les autres moyens de pêche habituels.

\section{Améliorations de la reproduction.}

Si l'amélioration de la production par une retenue partielle est possible, elle constituerait la meilleure amélioration envisageable pour la reproduction : il suffit d'exploiter la partie de la retenue maintenue à niveau sensiblement constant en étang d'alevinage.

Au cas où le concessionnaire n'aurait accepté qu'une atténuation des variations de niveaux dans la retenue partielle, atténuation intéressante dans la mesure où elle permet l'implantation d'une végétation aquatique (probablement de l'ordre de 1 mètre), il y aurait lieu de s'efforcer d'obtenir un niveau constant pendant trois semaines lors de la fraie du Brochet et pendant le mois de Juin, époque à laquelle l'eau non renouvelée pourra s'échauffer suffisamment sur les bords pour permettre la fraye des Cyprinides.

A ce point de vue, la moindre surface d'eau maintenue à niveau constant peut avoir un très grand intérêt pour peu que quelques pêcheurs veuillent bien reprendre des géniteurs dans la retenue et les remettre dans la retenue partielle à partir du jour où celle-ci est tout à fait close. Normalement, le niveau de cette surface d'eau sera le niveau légal, et il est rare que les rives de la retenue à pleins bords ne soient pas herbeuses; si elles ne le sont pas, il faudrait y poser des touffes d'herbes.

Faute de disposer d'une surface d'eau de niveau constant la repro- 
duction peut être améliorée très efficacement par des frayères artificielles flottantes. Les plus simples peuvent être constituées par des branchages feuillus au mois de Juin et par des branches de résineux et de genêts pour le Brochet, en Février-Mars. La difficulté est de placer ces frayères là où l'eau est la plus chaude sans risque d'assec. Si la retenue reçoit des sources dont la température soit de 8 à $10^{\circ}$ c'est près de celles-ci que les Brochets viendront frayer. Pour les Cyprinides, si la retenue est au sud ou à l'ouest du barrage et s'il y a une assez grande distance entre la prise d'eau et le barrage, c'est le long du barrage que les branchages formant les frayères seront le mieux placés et, en cet endroit, leur arrimage sera facile et l'assec aucunement à craindre.

\section{AmÉnagement dU BarRage.}

Etant donné la difficulté d'accès habituelle du fond de la retenue au pied amont du barrage : rives abruptes, rochers, vase..., on ne peut envisager la reprise du poisson lors des vidanges qu'à l'aval du barrage. Pour que cette reprise soit possible, il faut que le barrage réponde aux conditions suivantes :

Si les vannes de fond sont dans le barrage, elles doivent être à moins de 2 mètres du fond et le batardeau, qui a été édifié à l'amont du barrage pour permettre sa construction, doit être largement ouvert jusqu'au fond. D'autre part, la ou les conduites d'évacuation à travers le barrage doivent être inclinées régulièrement de 1 a $3 \%$. Si les vannes de fond ont été installées dans le tunnel de dérivation, le seuil de celui-ci doit être à moins de 2 mètres du fond de la retenue et le seuil des vannes au ras du radier du tunnel.

Les conditions nécessaires à l'évacuation du poisson à l'aval étant remplies, il faut encore que la disposition du canal de fuite des vannes de fond soit propice à la capture du poisson. Autant que faire se peut, il faudrait que ce canal de fuite soit constitué par un chenal bétonné, de section rectangulaire assez large pour que le niveau de l'eau n'y dépasse pas 60 centimètres lorsqu'il ne reste plus que 5 à 6 mètres d'eau dans la retenue et que les vannes sont grandes ouvertes. La longueur de ce canal doit être d'au moins 10 mètres pour une petite retenue et d'au moins 20 mètres pour une grande retenue. A l'extrémité de ce canal, il suffit que des trous d'une vingtaine de centimètres de profondeur aient été aménagés dans son radier pour pouvoir y placer une grille de peche retenue par des barres à mines fichées dans ces trous. Ces derniers peuvent être espacés de 1 mètre les uns des autres et légèrement inclinés vers l'aval pour faciliter le nettoyage de la grille au râteau lors de la vidange. Si les éventualités de vidange obligent à envisager un canal de fuite de plus de 1 mètre de profondeur, il serait souhaitable que les bajoyers de celui-ci comportent une ou plusieurs banquettes pour en faciliter l'accès.

Les vannes de fond et leur canal de fuite permettant la reprise du poisson, il faut encore que celui-ci soit conservé vivant. Or, quelles que soient les précautions prises pour la vidange, le poisson est toujours plus 


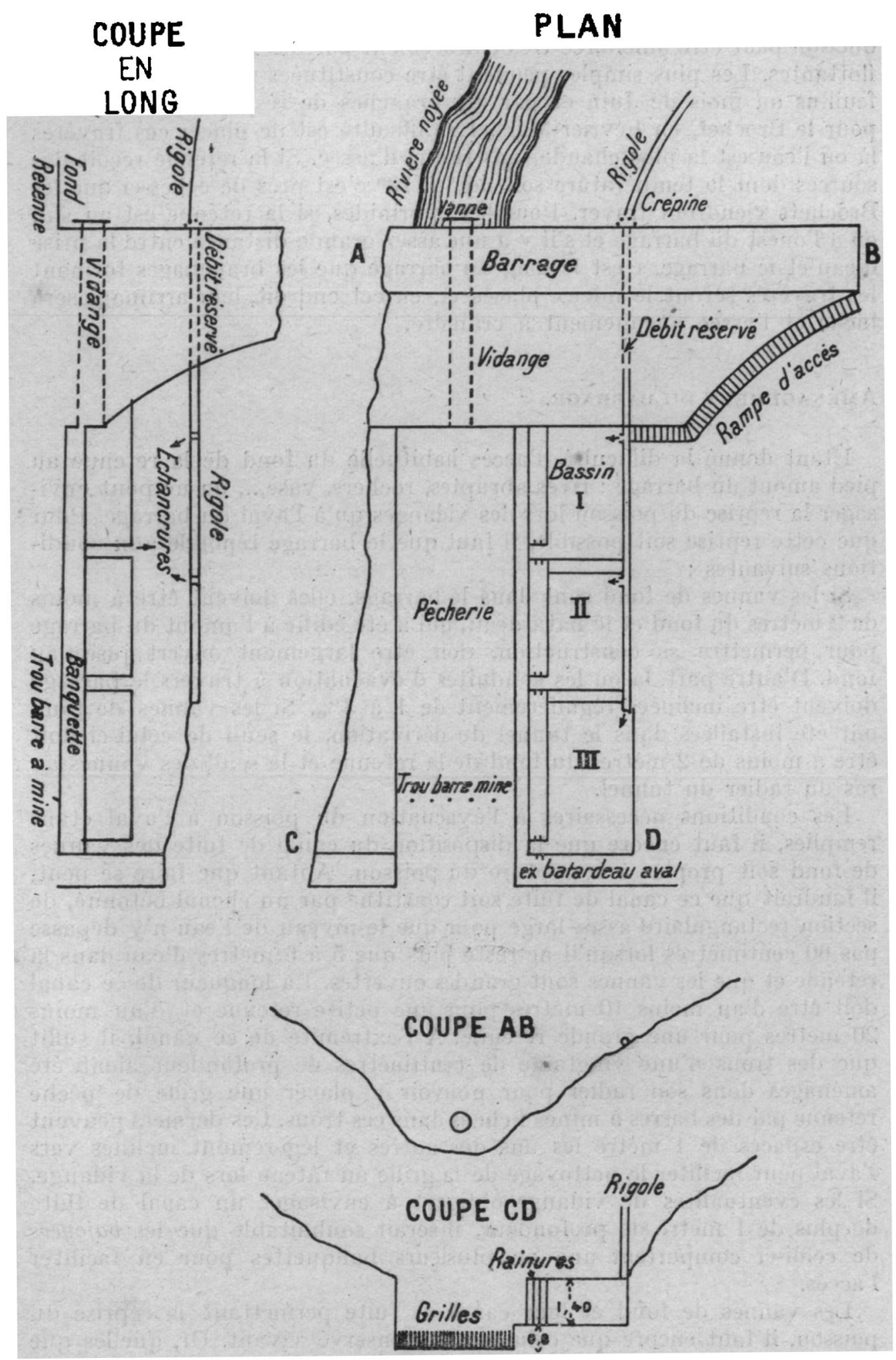

Aménagement à l'aval d'un barrage d'une pêcherie et de bassins alimentés par de l'eau captée à l'amont 
ou moins asphyxié lorsqu'il se laisse descendre dans l'eau boueuse de fin de vidange.

La seule solution pour conserver vivant ce poisson capturé au fond d'une gorge toujours peu accessible est de disposer à proximité immédiate d'un volume d'eau claire et oxygénée au moins vingt fois plus important que celui du poisson s'il s'agit de Cyprinides, et cent fois plus important pour la 'Truite, au cas où cette eau ne pourrait pas être renouvelée.

Les bassins pour emmagasiner cette eau peuvent être aménagés à peu de frais lors de la construction du barrage en utilisant le barrage luimême; un bajoyer du canal de fuite et éventuellement le batardeau de construction aval. Peu importe leur forme, mais il est souhaitable qu'il y en ait deux ou trois de 0,80 à 1 mètre de profondeur vidangeables séparément. S'il est possible que ces bassins soient alimentés constamment en eau pendant la fin de la vidange et après celie-ci, ils devraient comporter chacun une brèche de vidange sur toute leur hauteur. Deux paires de rainures de part et d'autre de la brèche permettront la pose d'une grille et la pose d'un batardeau en planche de façon à filtrer l'eau et régler son niveau dans chaque bassin.

Suivant les cas, une alimentation permanente des bassins sera facile, difficile ou impossible. Facile, lorsqu'une source, un ruisseau, ou plus fréquemment un canal d'amenée d'eau dans la retenue passe à flanc de vallée au-dessus des bassins : une prise d'eau aussi simple que possible et une rigole grossièrement tracée permettront d'alimenter les bassins en eau fraîche.

Difficile, mais cependant réalisable, quand le cours d'eau barré possède un affluent à peu de distance à l'amont du barrage; il faut qu'une conduite d'une dizaine de centimètres de diamètre ait été aménagée à travers Ie barrage, lors de sa construction, 4 ou 5 mètres au-dessus du fond de la retenue et au ras du sol du même côté que l'aflluent. Lors de la vidange, il suffit de barrer grossièrement l'affluent en question à une hauteur plus ou moins grande suivant son éloignement et dériver son eau, ou seulement une partie de celle-ci, vers l'orifice préexistant dans le barrage au moyen d'une rigole creusée dans le flanc de la retenue. Il est à remarquer que cet orifice peut avoir été imposé pour l'écoulement du débit réservé; dans ce cas, une crépine devra protéger son entrée.

Que la source d'eau soit à l'amont ou à l'aval de la rivière barrée, elle est forcément sur une rive et c'est sur cette rive que doivent être situés les bassins pour ne pas être obligé d'avoir recours à des tuyaux. Souvent, et parfois à juste titre, le concessionnaire compte sur les infiltrations à travers le barrage pour assurer le débit réservé. Le barrage fut-il en terre, cette eau est toujours désoxygénée et par conséquent impropre à l'alimentation des bassins.

L'alimentation permanente des bassins en eau peut être impossible; dans ce cas, il faut prévoir leur remplissage par l'eau de la vidange; pas tout à fait au début, car la première eau étant l'eau du fond, elle peut être désoxygénée, ni trop à la fin, car la vase désoxygène l'eau encore plus vite qu'elle ne la salit. Pratiquement, les brèches d'ouverture des bassins doivent ètre ouvertes sur le canal de fuite et si, comme il est probable, canal de fuite et bassins sont noyés au début de la vidange, on se 
contentera de fermer les bassins sitôt que le niveau de l'eau à l'aval le permettra.

Nous avons dit que lorsque l'eau ne peut pas être renouvelée dans les bassins, il faut donner à ceux-ci un volume au moins égal à 20 fois ou à 100 fois celui du poisson suivant qu'il s'agit de Cyprinides ou de Salmonides, mais qui pourra prévoir le volume (sensiblement égal au poids) du poisson? Certainement pas les pecheurs à la ligne qui probablement le surestimeront. On peut compter entre 10 et 100 kilogrammes à l'hectare pour les Cyprinides et 5 à 50 kilogrammes à l'hectare pour les Salmonides suivant la végétation aquatique (en proportion directe), l'altitude et l'acidité de l'eau (en proportion inverse).

Si les bassins peuvent etre alimentés par un débit permanent de l'ordre de quelques litres par minute, la taille des bassins peut être réduite de 3 et 4 fois, et par un débit de plusieurs dizaines de litres par minute de l'ordre de 10 fois.

\section{PÊche D'une retenue par vidange.}

Nous terminerons cet exposé par les mesures que nous estimerions devoir etre prises pour mener aussi bien que possible une vidange de retenue dans les conditions actuelles.

Un concessionnaire nous ayant avisé de son projet de vidanger sa retenue pour ravaler le barrage, nous lui demandons d'avancer cette vidange au printemps ou de la retarder à l'automne dans la mesure du possible, de nous communiquer le profil en long de cette retenue avec indication de la tranche turbinée habituellement et de nous faire connaître le délai auquel il peut consentir pour vidanger le "matelas ".

$1^{\circ}$ Compte tenu de ces renseignements et en accord avec les Ponts et Chaussées, nous proposons au Préfet de prendre un arrêté réglementant la vidange :

«A partir de telle date et de telle heure, la vitesse d'abaissement du niveau $a$ au niveau $b$, sera de $x \mathrm{cms}$ environ à l'heure, puis du niveau $b$ au niveau $c$, sera de $y$ cms environ à l'heure, puis du niveau $c$ au niveau $d$, sera de $z$ cms environ à l'heure.

Lorsqu'il ne restera que 4,5 ou 6 mètres d'eau, la manœuvre des vannes sera commandée par l'agent des Eaux et Forêts qui ne devra jamais interrompre la vidange plus d'une heure (pour éviter un assec à l'aval et une remontée du niveau dans la retenue).

Le concessionnaire, qui aura épuisé la tranche utile du barrage à l'amont, en même temps que celle du barrage à vidanger, fermera le barrage amont sitôt que celui-ci sera capable d'emmagasiner toute l'eau de la rivière jusqu'à deux heures après la fin de la vidange.

Le concessionnaire supportera la présence et l'activité de pêche de l'acheteur du poisson et de ses employés, et il assurera dans la mesure du possible, l'éclairage du canal de fuite et du fond de la retenue, et l'alimentation en eau des bassins.

Lors de la fermeture du barrage, les vannes devront laisser couler la 
moitié du débit de la rivière pendant un jour, le quart pendant quatre jours, puis le débit réservé \%.

$2^{\circ}$ Nous entrerons en contact avec le Directeur des Domaines pour lui demander de procéder à l'adjudication au profit de l'État du poisson qui pourra être pêché lors de la vidange. Cette adjudication ne pouvant être faite qu'au prix d'achat du kilo de poisson pêché par l'adjudicataire lui-même. Normalement, tant pour l'intérêt de la reprise du poisson que pour son écoulement, c'est la Société de pèche ayant le droit de pêche dans la retenue qui sera de beaucoup la mieux placée pour s'intéresser à cette adjudication. Si c'est elle ou la Fédération qui se porte adjudicataire nul doute que Domaines, Eaux et Forêts et Concessionnaire ne lui facilitent la reprise du poisson et même éventuellement, la conservation des poissons intéressants.

$3^{\circ}$ Il est inutile d'envoyer les gardes et de déplacer les gendarmes pour le jour $\mathrm{J}$ et l'heure $\mathrm{H}$ à laquelle l'abaissement de la retenue doit atteindre la "cote de fléchissement " (le niveau " a " ci-dessus). Il suffitde téléphoner quelques heures après pour s'assurer que la vitesse d'abaissement est bien conforme à l'arrêté préfectoral, et, tant que l'abaissement de l'eau n'a pas découvert un ouvrage d'art noyé ou la moitié de la surface mouillée au niveau $a$, il n'y a aucun autre contrôle à exercer que celui de vitesse de l'abaissement.

Si un vieux pont ou un barrage de moulin est sur le point d'être exondé, il faut y envoyer des gardes et l'adjudicataire, car du poisson peut y être surpris par l'assec ou seulement braconné. Si sa capture y est possible, il faut même y procéder immédiatement car c'est autant de poisson récupéré, poisson qui, par la suite, pourrait être perdu pour tout le monde.

Sitôt que les Ablettes commencent à piper, il faut s'assurer que les consignes pour le barrage amont sont respectées et inviter l'adjudicataire à senner ces Ablettes s'il dispose d'un filet ad hoc; il suffit d'une senne peu plombée à maille fine de 1 à 2 mètres de hauteur. Si elle ne sont pas sennées, ces Ablettes périront quasi-irrémédiablement et constitueront un ruban argenté de niveau sur le bord de la retenue : le concessionnaire ou l'adjudicataire devront engager des frais pour les ensevelir.

$4^{0}$ Avec l'aide du concessionnaire, l'adjudicataire aura fait le nécessaire pour disposer des bassins et les alimenter en eau dans la mesure du possible.

Sitôt la hauteur de l'eau dans le canal de fuite inférieure à 60 centimètres, il faut disposer des grilles en fer ou en bois dont l'intervalle, variable suivant les possibilités, peut être porté jusqu'à 3 centimètres.

$5^{\circ}$ Sitôt que le poisson commence à se laisser descendre vers l'aval, il sera nécessaire de mobiliser toutes les forces de police possibles pour tenir au loin tous les curieux tant à l'aval qu'à l'amont du barrage. On ne peut s'imaginer la violence du vieil instinct qui se réveille chez les spectateurs les plus pondérés : nous en avons vu se précipiter tout habillés dans l'eau vaseuse d'un fond de retenue, jusqu'au cou, pourtant agrémenté d'un col et d'une cravate, pour attraper une Carpe qu'ils n'auraient pas voulu payer 200 francs. 
$6^{\circ}$ Après la pêche, il faudra envoyer les gardes avec l'adjudicataire reconnaitre le fond de la retenue, car il se peut que celui-ci présentant une contre-pente, du poisson y ait été surpris par l'assec. Après enlèvement ou ensevelissement du poisson ainsi surpris, une tranchée devra etre ouverte dans la contre-pente pour que cette perte ne se renouvelle pas à la prochaine vidange.

70 Enfin, les gardes devront reconnaitre le cours aval de la rivière pendant deux ou trois jours après la fin de la vidange pour reconnaître la quantité de poissons morts et, après la fermeture du barrage, pour vérifier les consignes de fermeture échelonnée et pour s'opposer au braconnage dans la rivière mise peu à peu à sec.

$8^{\circ}$ En ce qui concerne l'alevinage ultérieur il faudra tenir compte du tonnage de poisson ayant vécu dans la retenue, de la quantité qui peut y avoir survécu, de la capacité de la rivière à l'amont en alevins des espèces voulues et du goût des pêcheurs.

S'il s'agit, comme il est fréquent, d'une retenue à poissons blancs située sur une rivière de première catégorie et qu'aucun poisson ne puisse y ètre demeuré vivant, en appelant $T$ le nombre de kilos de poissons qui y vivaient, la formule de lancement pourrait être la suivante:

$4 \mathrm{~T}$ alevins de Gardons ou de Rotengles de 2 ans $(5$ à $8 \mathrm{~cm}$.) ou plus;

$2 \mathrm{~T}$ alevins de Tanches;

$0,5 \mathrm{~T}$ alevins de Brêrnes;

$0,2 \mathrm{~T}$ alevins de Carpes, si les pècheurs le désirent.

L'année suivante il y aurait lieu d'immerger $1 \mathrm{~T}$ poignards-brochets ou $0,8 \mathrm{~T}$ poignards $+2 \mathrm{~T}$ civelles si un barrage limite leur remontée vers l'amont et si les pècheurs sont de cet avis. D'autre part, s'il ne semble pas qu'il y ait eu possibilité de frai naturel, il y aurait lieu de procéder a un deuxième lancement égal à la moitié du premier.

C'est volontairement que nous n'avons pas fait figurer la Perche dans cette formule de lancement. La Perche pondant en eau profonde, soit sur les éboulis, soit surtout dans les branches des arbres noyés, il est fréquent qu'elle pullule dans les retenues hydro-électriques et de ce fait y est atteinte d'un nanisme qui lui enlève tout intérêt. 